LETTERS

\title{
Palmar fasciitis and polyarthritis as a paraneoplastic syndrome associated with tubal carcinoma: a case report
}

\author{
D Denschlag, E Riener, P Vaith, C Tempfer, C Keck
}

Ann Rheum Dis 2004;63:1177-1178. doi: 10.1136/ard.2003.015693

A 73 year old white woman was admitted to our hospital in May 2003 complaining of lower abdominal pain for 1 month. On admission, the gynaecological examination disclosed a large, non-tender lower abdominal mass of $7 \mathrm{~cm}$ in diameter, which was highly suspicious of ovarian cancer with peritoneal infiltration in the computed tomography scan. In accordance with these findings, serum levels of CA 12-5 were about $3000 \mathrm{U} / \mathrm{ml}$ (normal range $<35 \mathrm{U} / \mathrm{ml}$ ).

Therefore, we performed an exploratory laparotomy. The frozen section of the adnexal mass showed a serous papillary carcinoma of fallopian tube origin. Consecutively, a complete staging with hysterectomy, bilateral salpingo-ophorectomy, omentectomy, and resection of the descending part of the colon due to tumour infiltration was performed.

Recovery was complicated owing to prolonged ileus-like symptoms. First line chemotherapy with topotecan in combination with carboplatin under study conditions (prospective, open label, phase II study) was started 3 weeks after the operation.

Before the diagnosis of carcinoma of the fallopian tube, the patient reported a progressive painful swelling and stiffness of both hands, especially the palms, which were noted by the patient to have thickened progressively since December 2002, with no improvement after initial corticosteroid treatment. Apart from generalised arthrosis, she denied any previous joint diseases, Raynaud's phenomenon, skin tightening, or trauma.

Physical examination on admission was remarkable for symmetrical swollen hands and fingers, painful on active and passive movement, and palmar fascial thickening with erythema (fig 1). The fingers of the patient showed flexion contractures; making a fist was impossible. No evidence of skin sclerosis or arthritis in other locations was found.

The patient's symptoms were orally treated with a cyclooxygenase(COX)-2 inhibitor, a transdermal delivery system for buprenorphine, and local ointments with diclofenac.

Beside an increased acute phase reaction (postoperatively), the immunological laboratory investigation failed to show any specific abnormalities (rheumatoid factor, antinuclear antibodies, antistreptolysin titre, antineutrophil cytoplasmic antibodies, complement $\mathrm{CH}_{50}$ and $\mathrm{C} 3 \mathrm{~d}$ ). Radiography of both hands showed a mild arthrosis in the joints, but no signs of acute arthritis.

After two cycles of antineoplastic treatment the patient's paraneoplastic symptoms currently show a good response: the fasciitis and acute arthritis have gradually improved, but the contractures in both hands persist despite extensive physiotherapy.

\section{DISCUSSION}

Paraneoplastic syndromes affect a variety of organ systems and are often an initial sign of occult malignancies. About 30 cases have been reported describing palmar fasciitis and polyarthritis (PFPA)-like changes of the hands in association with malignant tumours. PFPA has been reported to be

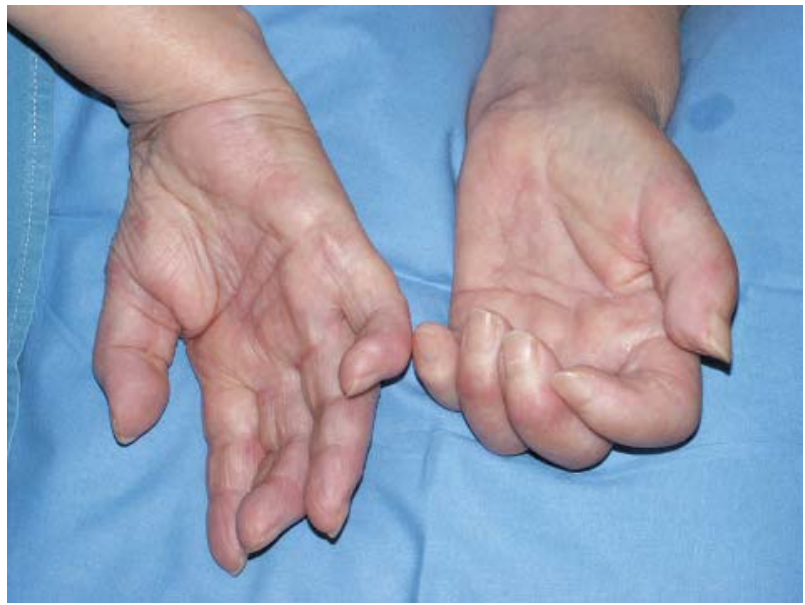

Figure 1 Both hands of the patient with fingers in maximal extension, showing contractures and nodular palmar fascial thickening.

associated with different neoplasms-for example, ovarian cancer, ${ }^{1}$ carcinoma of the breast, ${ }^{2}$ carcinoma of the prostate, ${ }^{3}$ and gastric tumours ${ }^{4}$ preceding or accompanying the diagnosis of malignancy.

In our case we present a patient demonstrating a severe progressive deforming rheumatic disease affecting the palms and fingers of both hands, with the onset of the corresponding symptoms 5 months before the diagnosis of a malignant neoplasm was made.

To our knowledge this is the first report of the association of PFPA as a paraneoplastic syndrome with a carcinoma of the fallopian tube.

The underlying immunological mechanisms have not been defined so far and may result from activation of certain factors with profibrotic activities-for example, transforming growth factor $\beta$ or connective tissue growth factor. ${ }^{6}$ As long as the pathomechanisms of PFPA are unclear, the only effective therapy is cancer treatment, which may lead to arrest or even complete resolution of the rheumatic disease. $^{7}$

The characteristic hand deformities of PFPA, recently described with the illustrative term of woody hands, ${ }^{7}$ should alert the clinician to search for an underlying malignant disease.

\section{Authors' affiliations \\ D Denschlag, E Riener, P Vaith, C Tempfer, C Keck, University of Freiberg School of Medicine, Germany}

Correspondence to: Dr D Denschlag, Hugstetter Str 55, Freiburg, 79106 Germany; ddensch|@frk.ukl.uni-freiburg.de

Accepted 20 October 2003 


\section{REFERENCES}

1 Medsger TA, Dixon JA, Garwood VF. Palmar fasciitis and polyarthritis associated with ovarian carcinoma. Ann Intern Med 1982:96:424-31.

2 Valverde-Garcia J, Juanola-Roura X, Ruiz-Martin JM, Nolla-Sole JM, Rodriguez-Moreno J, Roig-Escofet D. Paraneoplastic palmar fasciitispolyarthritis syndrome associated with breast cancer. J Rheumatol 1987; 14:1207-9.

3 Van den BL, Vanneste SB, Knockaert DC. Palmar fasciitis and arthritis associated with cancer of the prostate. Acta Clin Belg 1991;46:106-10.
4 Chakravarty E, Genovese MC. Rheumatic syndromes associated with malignancy. Curr Opin Rheumatol 2003;15:35-43.

5 Enomoto M, Takemura H, Suzuki M, Yuhara T, Akama T, Yamane K, et al. Palmar fasciitis and polyarthritis associated with gastric carcinoma: complete resolution after total gastrectomy. Intern Med 2000;39:754-7.

6 Ihn H. Pathogenesis of fibrosis: role of TGF-beta and CTGF. Curr Opin Rheumato 2002;14:681-5.

7 Alexandroff $\mathrm{AB}$, Hazleman BL, Matthewson M, Black CM, Rytina E, Crawford R, et al. Woody hands. Lancet 2003;361:1344.

\title{
Mycobacterium chelonae finger infection associated with Raynaud's phenomenon
}

\author{
H Sari-Kouzel, P R Chadwick, L T S W Muir, A L Herrick, D W Denning
}

Ann Rheum Dis 2004;63:1178-1179. doi: 10.1136/ard.2003.009506

$M$ ycobacterium chelonae infections are uncommon and have not been reported as a complication of Raynaud's phenomenon previously. We describe a patient with this problem who responded well to treatment.

\section{CASE REPORT}

A 21 year old female telephonist was referred with a 1 week history of swollen right index and middle fingers. There was no history of trauma but she had had Raynaud's phenomenon since the age of 12 . There was no history of recent travel abroad or of unusual hobbies.

On examination she was apyrexial, she had diffuse swelling but no discolouration of the right index and middle fingers. She had no blisters or ulceration and the fingers were not tender to touch. Nine days later, despite flucloxacillin orally and a non-steroidal anti-inflammatory agent, all her fingers were cold and bluish, and the right index and right middle fingers remained diffusely swollen. She was admitted for intravenous prostacyclin because of worsening of her Raynaud's phenomenon.

Her baseline investigations were normal, including erythrocyte sedimentation rate and $\mathrm{C}$ reactive protein. A hand $x$ ray examination showed no bony abnormality. Blood cultures were negative. Her immunoglobulin levels were normal, and cryoglobulins were not detected. Her autoantibody profile was negative except for raised IgG cardiolipin antibodies at 46 GPLU (reference range 0-13).

As she finished the 72 hour intravenous prostacyclin infusion, a pustule appeared on the pulp of the right index finger, and intravenous ceftriaxone was started. Two days later a second pustule appeared on the other finger (fig l). Microscopy of a needle aspiration showed acid fast bacilli. Antibiotics were changed to clarithromycin $500 \mathrm{mg}$ twice daily and ciprofloxacin $750 \mathrm{mg}$ twice daily. Both lesions were incised and drained, and histology of the material showed an abscess wall with no granulomata.

$M$ chelonae was cultured from pus and was susceptible in vitro to azithromycin and clarithromycin, but resistant to other antituberculous agents, as well as to ciprofloxacin. As her lesions were healing at the time these results became available (14 days later) her antibiotics were not changed.

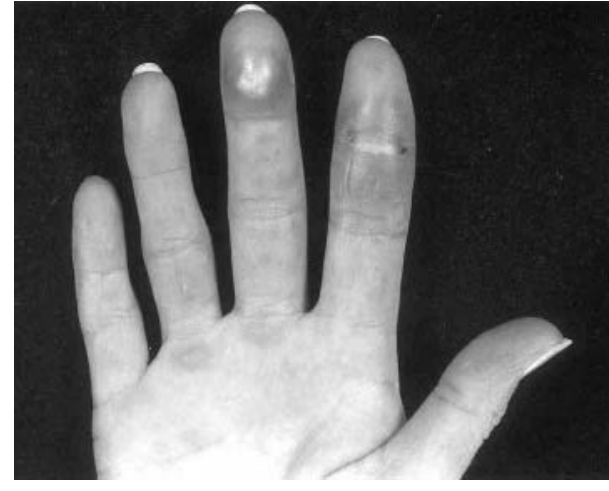

Figure 1 Pustules on the index and middle fingers of the right hand.

She continued the combination for a total of 5 weeks and her fingers healed completely.

\section{DISCUSSION}

$M$ chelonae is associated with a variety of infections. Cutaneous lesions occur secondary to wound infections after surgery, accidental trauma, or needle injections. ${ }^{12} M$ chelonae isolates are susceptible in vitro to clarithromycin, but generally resistant to ciprofloxacin. The use of at least two drugs (one of which should be clarithromycin) is recommended for treatment of $M$ chelonae infection, as the emergence of resistance is a risk associated with monotherapy. ${ }^{3}$ Treatment is usually given for 4-6 months, but our patient responded to combination therapy within weeks. A case has been reported of postoperative infection at a donor vein graft site that healed after 2 months of clarithromycin treatment, combined with heat treatment. ${ }^{5}$ Thus shorter courses of treatment may be appropriate in non-immunosuppressed patients.

Isolates of $M$ chelonae have optimal growth at $28-30{ }^{\circ} \mathrm{C},{ }^{6}$ unlike the standard $35^{\circ} \mathrm{C}$ for most organisms. Our patient had severe Raynaud's disease before the infection, so the coldness of her hands may have promoted the growth of this unusual organism. We are not aware of any previous reports of 
$M$ chelonae infection in a patient with Raynaud's phenomenon. A significant portion of the 100 patients with $M$ chelonae infections reported by Wallace et al were receiving corticosteroids or immunosuppressant drugs, or both. In their series 35 patients had a localised cutaneous infection and only five had no history of trauma, of whom three had an underlying immunosuppressive condition.

Our patient had no history of trauma, she was immunocompetent, but had a history of Raynaud's phenomenon. Because there were no systemic features during the acute phase of her illness, an atypical infection was suspected and acid fast bacilli were specifically looked for. In conclusion, unusual presentations have unusual explanations and should raise suspicion of atypical infections.

\section{ACKNOWLEDGEMENTS}

Mycobacterial identification and susceptibility tests were performed at Newcastle Public Health Laboratory, Newcastle, UK. We thank John Magee of Newcastle PHL for helpful discussions of this case.

\section{Authors' affiliations}

H Sari-Kouzel, A L Herrick, University of Manchester Rheumatic Diseases Centre, Hope Hospital, Salford M6 8HD, UK

P R Chadwick, Department of Microbiology, Hope Hospital, Salford M6 8HD, UK

L T S W Muir, Department of Orthopaedic Surgery, Hope Hospital, Salford M6 8HD, UK
D W Denning, Research and Teaching Block, University of Manchester, Wythenshawe Hospital, Southmoor Rd, Manchester M29 9LT, UK

Correspondence to: Dr H Sari-Kouzel, Department of Rheumatology, Basildon and Thurrock University Hospital, Nethermayne, Basildon, Essex SS16 5NL, UK; hsarikouzel@hotmail.com

Accepted 17 October 2003

\section{REFERENCES}

1 Brown BA, Wallace RJ. Infections due to non tuberculous mycobacteria. In: Mandell GL, Bennett JE, Dolin R, eds. Mandell, Douglas, and Bennett's principles and practice of infectious diseases, 5th ed. Philadelphia: Churchill Livingstone, 2000:2630-5.

2 Zenone T, Boibieux A, Tigaud S, Fredenucci JF, Vincent V, Chidiac C, et al. Non-tuberculous mycobacterial tenosynovitis: a review. Scand J Infect Dis 1999;31:221-8.

3 Tebas P, Sultan F, Wallace RJ Jr, Fraser V. Rapid development of resistance to clarithromycin following monotherapy for disseminated Mycobacterium chelonae infection in a heart transplant patient. Clin Infect Dis 1995;20:443-4.

4 Driscoll MS, Tyring SK. Development of resistance to clarithromycin after treatment of cutaneous Mycobacterium chelonae infection. J Am Acad Dermatol 1997:36:459-6.

5 Engelhardt E, Feldmann R, Skaria A, Salomon D. Postoperative infection with Mycobacterium chelonae. Hautarzt 1996;47:863-6.

6 Wallace RJ Jr. Recent changes in taxonomy and disease manifestations of the rapidly growing mycobacteria. Eur J Clin Microbiol Infect Dis 1994; 13:953-60.

7 Wallace RJ Jr, Brown B, Onyi GO. Skin, soft tissue, and bone infections due to Mycobacterium chelonae chelonae: importance of prior corticosteroid therapy, frequency of disseminated infections, and resistance to oral antimicrobials other than clarithromycin. J Infect Dis 1992;166:405-12.

\title{
Progression of lupus nephritis during treatment with mycophenolate mofetil
}

\author{
K Ahmadi-Simab, P Lamprecht, W L Gross
}

$\mathrm{L}$ upus nephritis (LN) determines the prognosis of systemic lupus erythematosus. The standard treatment for the proliferative forms (focal and diffuse proliferative LN, WHO classes III and IV) is intravenous pulse cyclophosphamide in combination with oral prednisone (Austin scheme). ${ }^{12}$ Recently mycophenolate mofetil (MMF) has been shown to be as effective as standard pulse cyclophosphamide for the induction of remission in proliferative $\mathrm{LN}(\mathrm{n}=2 \mathrm{l}$ in each treatment group). Side effects of MMF were fewer than with pulse cyclophosphamide. ${ }^{3}$ We report on a patient with deteriorating renal function and switch of LN class despite MMF treatment.

\section{CASE REPORT}

Systemic lupus erythematosus was diagnosed in a 63 year old white patient with non-erosive polyarthritis, photosensitivity, malar rash, nephritic sediment (250 erythrocytes/high powered field, dysmorphic erythrocytes, non-selective glomerular proteinuria $2.26 \mathrm{~g} /$ day), antinuclear antibodies, and antidsDNA antibodies. A renal biopsy disclosed non-proliferative LN with mesangial hypercellularity (WHO class type IIb). The patient had experienced adverse effects with azathioprine and methotrexate previously. Thus, MMF (2.0 g/day p.o., CellCept, Roche Pharmaceuticals) and prednisolone $(1.0 \mathrm{mg} /$ day p.o. with subsequent tapering) were given. However, renal function deteriorated within 3 months, with declining creatinine clearance $(65 \mathrm{ml} / \mathrm{min}$ to $49.1 \mathrm{ml} / \mathrm{min})$, increasing serum creatinine $(112 \mu \mathrm{mol} / \mathrm{l}$ to $155 \mu \mathrm{mol} / \mathrm{l})$, and persistent nephritic sediment and proteinuria. A second renal biopsy disclosed a switch to focal proliferative LN (WHO class III). Treatment with MMF was discontinued and intravenous cyclophosphamide pulses were given, resulting in stabilised renal function and reduced proteinuria ( $0.6 \mathrm{~g} /$ day $)$ after six cyclophosphamide pulses.

\section{DISCUSSION}

In this case MMF was given in non-proliferative mesangial LN (WHO class IIb), characterised by a better prognosis than proliferative LN. However, renal function deteriorated owing to progression of the LN to focal proliferative LN (WHO class III). Although the presence of both LN types at onset and late effects of MMF on renal function after switching to pulse cyclophosphamide cannot be ruled out, the course with deteriorating renal function during MMF treatment suggests 
inefficacy of MMF and a concomitant switch of LN class in this case. A personal communication on a higher relapse rate with MMF than with cyclophosphamide pulse for proliferative LN during follow up of Chan's study 3 (46\% v 17\%; $\mathrm{p}=0.019)$ has been reported in a review recently. ${ }^{4}$

We conclude that further studies on larger patient groups are needed to establish the place of MMF in the treatment of LN and to develop therapeutic algorithms, including early intervention in non-responders.

\section{Authors' affiliations}

K Ahmadi-Simab, P Lamprecht, W L Gross, Department of Rheumatology, University Hospital of Schleswig-Holstein, Campus Luebeck, and Rheumaklinik Bad Bramstedt, Ratzeburger Allee 160, 23538 Luebeck, Germany
Correspondence to: Dr K Ahmadi-Simab, ahmadi@rheuma-zentrum.de; ahmadi@ik-h.de

Accepted 30 September 2003

\section{REFERENCES}

1 Austin HA 3rd, Klippel JH, Balow JE, le Riche NG, Steinberg AD, Plotz PH, et al. herapy of lupus nephritis. Controlled trial of prednisone and cytotoxic drugs. N Engl J Med 1986;314:614-19.

2 Boumpas DT, Austin HA 3rd, Vaughn EM, Klippel JH, Steinberg AD, Yarboro $\mathrm{CH}$, et al. ontrolled trial of pulse methylprednisolone versus two regimens of pulse cyclophosphamide in severe lupus nephritis. Lancet 1992;340:741-5.

3 Chan TM, Li FK, Tang CS, Wong RW, Fang GX, Ji YL, et al. Efficacy of mycophenolate mofetil in patients with diffuse proliferative lupus nephritis. N Engl J Med 2000;343:1 156-62.

4 Gescuk BD, Davis JC. Novel therapeutic agents for systemic lupus erythematosus. Curr Opin Rheumatol 2002;14:515-21.

\title{
Assessment of the clinically relevant change in pain for patients with sciatica
}

\author{
B Giraudeau, S Rozenberg, J-P Valat
}

Ann Rheum Dis 2004;63:1180-1181. doi: 10.1136/ard.2003.015792

$\mathrm{T}$ he visual analogue scale (VAS) is widely used for pain assessment. However, the minimum clinically relevant change remains a debated question, even with regard to the method of assessment. On the one hand, considering intra-individual change in acute pain, Todd et al suggested a crude change of $13 \mathrm{~mm}$ (for a $100 \mathrm{~mm}$ VAS) to discriminate between reporting a little less or a little more pain. ${ }^{1}$ In the study of Farrar et al the best cut off points were estimated at $-20 \mathrm{~mm}$ (for a crude change) or $-33 \%$ (for a relative change) to best discriminate between patients who require a rescue opioid dose or not. ${ }^{2}$ Similar results were also derived when considering patient self appreciation of improvement in chronic pain. ${ }^{3}$ On the other hand, Redelmeier et al suggested focusing on interindividual change to assess a minimal important difference. ${ }^{4}$ Wells et al thus observed that when a patient feels "somewhat worse" than another patient who faces him, then there is a $16 \%$ relative difference in their mean answer to a pain questionnaire.

We performed an ancillary study to a clinical trial in order to estimate the minimum clinically relevant change in pain for patients with sciatica. The main study was a randomised double blind trial conducted to assess the effectiveness of epidural corticosteroid injections in patients with sciatica, presumably due to a herniated nucleus pulposus, for 15180 days. ${ }^{6}$ We thus administered three epidural injections at 48 hour intervals with $2 \mathrm{ml}$ prednisolone acetate $(50 \mathrm{mg}$ ) or $2 \mathrm{ml}$ isotonic saline. Self evaluation was the primary outcome, measured at day 20 on a four item scale (recovery, important improvement, poor improvement, or worse). The first two items were then pooled, thus defining a treatment success, while the last two items defined a treatment failure. As a secondary outcome we assessed the severity of pain on a VAS both at day 0 and day 20. Eighty five patients were included in the main study. However, in the present work, analyses were performed on a subsample of 75 patients for

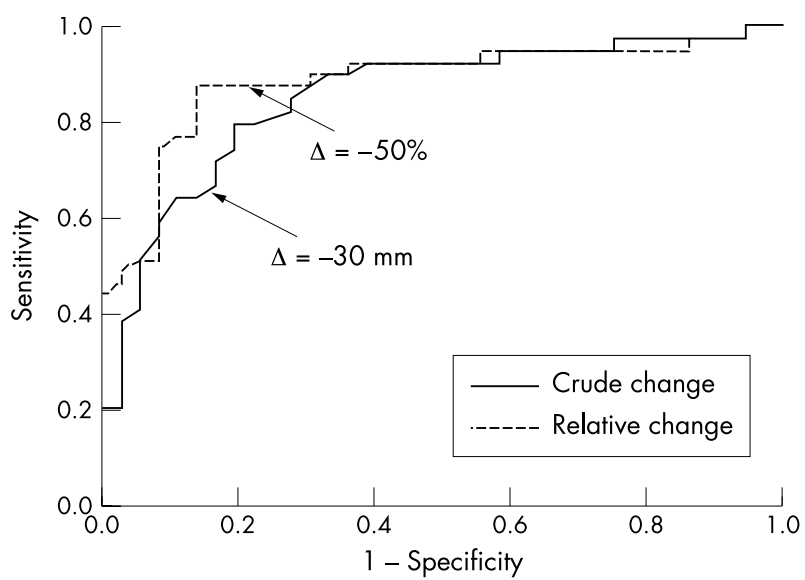

Figure 1 Receiver operating characteristic curves for crude and relative changes in pain assessment $v$ self evaluation. Self evaluation is defined as "treatment success" or "treatment failure" at day 20. Change in pain assessment is defined as the crude or relative change in pain VAS assessment between day 0 and day 20 .

whom we had both the self evaluation at day 20 and the two pain assessments. The subsample comprised 45 men and 30 women with a mean (SD) age of 41 (11) years. Those patients had had pain for a mean (SD) duration of 49 (41) days and their mean baseline pain was 58 (16). For 39 of those 75 patients, treatment was a success. We performed analyses without taking into account the randomisation arm: there is indeed no foundation for a different relationship between self evaluation and pain assessment according to a patient's treatment. For each patient, we calculated both the crude and relative changes in pain assessment and plotted receiver operating characteristic curves (fig 1). Table l shows the 


\begin{tabular}{|c|c|c|c|c|}
\hline & \multicolumn{4}{|c|}{ (A) Cut off value for crude change in pain VAS between day 0 and day 20} \\
\hline & $-40 \mathrm{~mm}$ & $-30 \mathrm{~mm}$ & $-20 \mathrm{~mm}$ & $-10 \mathrm{~mm}$ \\
\hline $\begin{array}{l}\text { Sensitivity } \\
\text { Specificity } \\
\text { Accuracy }^{*}\end{array}$ & $\begin{array}{l}56.4(39.6 \text { to } 72.2) \\
91.7(77.5 \text { to } 98.2) \\
73.3(61.9 \text { to } 82.9)\end{array}$ & $\begin{array}{l}76.9(60.7 \text { to } 88.9) \\
80.6(64.0 \text { to } 91.8) \\
78.7(67.7 \text { to } 87.3)\end{array}$ & $\begin{array}{l}87.2(72.6 \text { to } 95.7) \\
69.4(51.9 \text { to } 83.7) \\
78.7(67.7 \text { to } 87.3)\end{array}$ & $\begin{array}{l}92.3(79.1 \text { to } 98.4) \\
58.3(40.8 \text { to } 74.5) \\
76.0(64.7 \text { to } 85.1)\end{array}$ \\
\hline & \multicolumn{4}{|c|}{ (B) Cut off value for relative change in pain VAS between day 0 and day 20} \\
\hline & $-60 \%$ & $-50 \%$ & $-40 \%$ & $-33 \%$ \\
\hline $\begin{array}{l}\text { Sensitivity } \\
\text { Specificity } \\
\text { Accuracy* }\end{array}$ & $\begin{array}{l}76.9(60.7 \text { to } 88.9) \\
88.9(73.9 \text { to } 96.9) \\
82.7(72.2 \text { to } 90.4)\end{array}$ & $\begin{array}{l}87.2(72.6 \text { to } 95.7) \\
80.6(64.0 \text { to } 91.8) \\
84.0(73.7 \text { to } 91.4)\end{array}$ & $\begin{array}{l}87.2(72.6 \text { to } 95.7) \\
69.4(51.9 \text { to } 83.7) \\
78.7(67.7 \text { to } 87.3)\end{array}$ & $\begin{array}{l}89.7(75.8 \text { to } 97.1) \\
69.4(40.8 \text { to } 74.5) \\
80.0(69.2 \text { to } 88.4)\end{array}$ \\
\hline
\end{tabular}

performance indexes associated with several cut off points. Optimal cut off points, which thus offer the best compromise between sensitivity and specificity, are estimated at $-30 \mathrm{~mm}$ for the crude change and $-50 \%$ for the relative change. These values are higher than those estimated by Todd et al ${ }^{1}$ and Farrar et al. ${ }^{23}$ Such discrepancies may be due to a difference in the "gold standard". They may also be related to the length of the period of observation because we focused on a 3 week period.

In conclusion, the values we estimated may be seen as the clinically relevant long term changes in pain assessment for patients with sciatica. Such findings, however, have to be confirmed by further studies planned with this specific aim because patients included in a randomised trial may not be representative of the general population of patients with sciatica.

\section{Authors' affiliations}

B Giraudeau, INSERM CIC202, Faculté de Médecine, Tours, France S Rozenberg, Service de Rhumatologie, CHU de La Pitié, Paris, France J-P Valat, Service de Rhumatologie, CHU, Tours, France
Correspondence to: Dr B Giraudeau, INSERM CIC202, Faculté de Médecine, 2bis Bd Tonnellé, 37032 Tours cedex, France; giraudeau@ med.univ-tours.fr

Accepted 17 September 2003

\section{REFERENCES}

1 Todd KH, Funk KG, Bonacci R. Clinical significance of reported changes in pain severity. Ann Emerg Med 1996;27:485-9.

2 Farrar JT, Portenoy RK, Berlin JA, Kinman JL, Strom BL. Defining the clinical important difference in pain outcome measures. Pain 2000;88:287-94.

3 Farrar JT, Young JP, LaMoreaux L, Werth JL, Michael PR. Clinical importance of changes in chronic pain intensity measured on an 11-point numerical pain rating scale. Pain 2001;94:149-58.

4 Redelmeier DA, Guyatt GH, Goldstein RS. Assessing the minimal important difference in symptoms: a comparison of two techniques. J Clin Epidemiol 1996;49:1215-1219.

5 Wells GA, Tugwell P, Kraag GR, Baker PR, Groh J, Redelmeier DA. Minimum important difference between patients with rheumatoid arthritis: the patient's perspective. J Rheumatol 1993;20:557-60.

6 Valat JP, Giraudeau B, Rozenberg S, Goupille Ph, Bourgeois P, MicheauBeaugendre $V$, et al. Epidural corticosteroid injections for sciatica: a randomized, double blind, controlled clinical trial. Ann Rheum Dis 2003;62:639-43. 